\title{
Comparative analysis of the diagnostic value of several methods for the diagnosis of patent foramen ovale
}

\author{
Fangfang Liu ${ }^{1}$, Qingyu Kong ${ }^{2}$, Xiaojun Zhang ${ }^{2}$, Yan $\mathrm{Li}^{2}$, Shimin Liang ${ }^{2}$, Shuang $\mathrm{Han}^{2}$, and \\ Guishuang $\mathrm{Li}^{1}$ \\ ${ }^{1}$ Shandong University Qilu Hospital \\ ${ }^{2}$ Affiliation not available
}

October 19, 2020

\begin{abstract}
Objective: The aim of this research was to compare the sensitivity and positive predictive value of contrast transcranial Doppler (c-TCD), contrast- transthoracic echocardiography (c-TTE) and contrast- transesophageal echocardiography (c-TEE), to determine the best method for diagnosing patent foramen ovale $(\mathrm{PFO})$ and to provide a reference for the further improvement of clinical practice. Methods: We investigated 161 patients who suffered from migraines, cryptogenic stroke, TIA, and cerebral infarction of unknown cause. All patients underwent transcatheter examination, and the results of the right heart catheterization (RHC) were considered the gold standard for PFO diagnosis. Results: The present study revealed that c-TTE with the Valsalva maneuver had a higher sensitivity in detecting PFO related right-to-left shunt (PFO-RLS), but it might have a higher rate of misdetection than c-TCD. Conclusion: Patients with suspected PFO can be examined with c-TCD first, and if positive results are obtained, then c-TTE and c-TEE should be performed for further confirmation.
\end{abstract}

\section{Introduction}

In recent years, embolic strokes of undetermined source (ESUS) have emerged as a new clinical construct for characterizing cryptogenic stroke (CS). Most patients were young and have no medical history of hypertension, diabetes or hyperlipidemia, and routine imaging examination also failed to detect significant vascular abnormalities ${ }^{[1]}$. Currently, patent foramen ovale (PFO) has been reported to be strongly associated with a number of diseases, including cryptogenic stroke ${ }^{[2]}$, transient ischemic attack (TIA) ${ }^{[3]}$, migraine ${ }^{[4]}$, peripheral arterial embolism, and decompression sickness ${ }^{[5]}$.

Foramen ovale is a persistent fetal communication between the right and left atrium due to incomplete closure of the atrial septum. After birth, with the interruption of umbilical blood circulation and the establishment of respiration, the foramen ovale closes functionally, and complete closure generally occurs 5 to 7 months after birth. If the foramen ovale is still unclosed in children over 3 years old, PFO can be diagnosed. If the right atrial pressure is higher than left atrial pressure (for example, during cough, inspiration, or the Valsalva maneuver), blockages in the venous system can flow to the left atrium. Patent foramen ovale is the cause of $95 \%$ of paradoxical embolisms ${ }^{[5]}$.

There are three methods for diagnosing PFO: contrast-transcranial doppler(c-TCD), transthoracic echocardiography (TTE), and transesophageal echocardiography (TEE). TEE has a major role in the diagnosis of PFO. The diagnostic sensitivity of PFO related right-to-left shunt (PFO-RLS) with contrast transcranial Doppler (c-TCD) is similar to that with TEE. However, c-TCD has a limited ability to differentiate cardiac from pulmonary RLS. The use of contrast echocardiography was first reported by Gramiak ${ }^{[6]}$. Currently, contrast transthoracic echocardiography (c-TTE) with the Valsalva maneuver is extensively used for the 
detection and semiquantitative assessment of PFO-RLS ${ }^{[7]}$. However, the diagnostic criteria are not unified. Thus, the aim of this research was to compare the sensitivity and specificity of each examination method to determine which is the best method for diagnosing PFO and to provide a reference for the further improvement of clinical practice.

\section{Materials and Methods}

\section{Patient Population}

We investigated 161 patients ( 86 male, 75 female; mean age, $42.0 \pm 15.6$ years) admitted to the Departments of Neurology and Cardiology in Qilu Hospital, Shandong University from August 2018 to July 2019 who suffered from migraine, cryptogenic stroke, TIA, and cerebral infarction of unknown cause, including 55 migraine, 56 cryptogenic stroke, 20 cases of both symptoms, 19 cases of dizziness or syncope, 9 TIA cases, and 2 epilepsy cases. Exclusion criteria: (1) Cerebral embolism due to any cause, for example, cardiac cerebral embolism or arteriosclerosis; (2) Contraindications for antiplatelet or anticoagulant therapy, for example, due to severe bleeding in the past 3 months, severe retinopathy, history of intracranial hemorrhage or other intracranial disease; (3) Obstruction caused by inferior vena cava or pelvic venous thrombosis, systemic or local infection, septicemia, intracardiac thrombosis, etc.; (4) Pregnancy; (5) Concurrent pulmonary arterial hypertension or PFO that cannot be closed; (6) Acute stroke in the past 2 weeks.

The study was approved by the local ethics committee, and all patients or their relatives provided written informed consent to participate in this study prior to the examination.

\section{Saline contrast preparation}

The right anterior elbow vein was selected for the placement of an indwelling needle. The contrast agent was prepared by mixing it with $3 \mathrm{ml}$ of air, $30 \mathrm{ml}$ of saline solution, and 3-5 ml of the patient's blood and intensively mixing it back and forth 20 times between two 30-ml syringes connected by a T-branch pipe.

\section{c-TCD Examination}

TCD was performed using the Multi-DopX4 Transcranial doppler (DWL Electronic Systems, Sipplingen, Germany) with a $2 \mathrm{MHz}$ probe. Middle cerebral artery flow was monitored through the temporal bone window at a depth of 50-65 mm. The study was performed by a neurologist who specialized in this technique and was blinded to the results of the echocardiographic study. After value of the the middle cerebral artery blood flow velocity was obtained, the contrast agent was quickly injected via the established route of the anterior elbow vein. The contrast agent was prepared, and right-to-left shunt was diagnosed when TCD detected microsignals in the middle cerebral artery, in both the resting state and during performance of the Valsalva maneuver. The severity of the shunt was quantified as negative (no microsignals), mild (1-20 microsignals, 1-10 per side), moderate ([?]20 microsignals, ¿10 per side), or extensive (¿30 microsignals or rain curtain-like signals) (Figure 1).

\section{c-TTE and c-TEE Examination}

Contrast- TTE was conducted using a GE Vivid E9 or E95 platform equipped with a $3.7-5 \mathrm{MHz}$ M5S transducer (Horten, Norway) or a Philips EPIQ7 platform equipped with a 1-5 MHz S5-1 transducer (Philips USA). All patients were asked to remain still in the left lateral position. Conventional 2D echocardiography was performed to acquire the standard apical, parasternal, and subxyphoid four-chamber views. Color flow Doppler was used to determine whether there was RLS at the foramen ovale of the interatrial septum. First, with the patient in a resting state, the contrast agent was mixed and injected as the same manner that was used for c-TCD. The apical four-chamber view was maintained, and after microbubbles filled the right atrium, observation continued to determine whether microbubbles appeared in the left atrium. The operation was then repeated while the patient performed the Valsalva maneuver. When the right atrium was filled with microbubbles, the patient was instructed to immediately perform the Valsalva maneuver. RLS was graded according to the highest number of microbubbles observed in the left chamber in a single frame: image-negative (no microbubbles), mild (1-10 microbubbles), moderate (11-30 microbubbles), or extensive 
(¿30 microbubbles or left chamber opacification) (Figure 2). If microbubbles appeared in the left atrium within five cardiac cycles after they are released, RLS was primarily considered to be derived from a PFO. If microbubbles appeared in the left atrium after more than five cardiac cycles, RLS was assumed to originate from a pulmonary arteriovenous malformation.

Contrast- TEE was performed using the same system fitted with a 2-7 MHz multi-frequency transesophageal probe. To improve the tolerance of the test, pharyngeal topical anesthesia was administered using tetracaine hydrochloride gel $15 \mathrm{~min}$ before the examination. The probe was pushed in 30-40 cm, rotated within $45 \mathrm{deg}-$ 140deg to clearly display ascending aorta root, the septum primum and the septum secundum, and to observe whether an opened PFO existed in the two-dimensional view, measured PFO width and length at the same time. Color Doppler was used to observe whether there was a shunt across the PFO. Then, with the patient at rest and performing the Valsalva maneuver, prepared saline contrast agent was injected according to the procedure described above. To ensure maximal diagnostic yield, a standard apical four-chamber view was performed with the administration of contrast agents. The severity of the microbubbles was quantified as the same way as for c-TTE (Figure 3).

Right heart catheterization and transcatheter closure

All patients for whom RLS was indicated underwent transcatheter examination, and the results of the right heart catheterization (RHC) were regarded as the gold standard for PFO diagnosis. After localized anesthesia with lidocaine, the femoral vein was punctured with an intravenous cannula, and then a $6-\mathrm{F}$ sheath was inserted over the guidewire to reach the foramen ovale at the right atrium; if the catheter could pass though the foramen ovale to reach the left atrium, then PFO could be diagnosed. If the sheath failed to pass though the foramen ovale, then the guidewire was exchanged for a 0.035 -inch hydrophilic wire, and if the hydrophilic wire could pass though the foramen ovale to reach the left atrium, then PFO can also be diagnosed. Finally, if the hydrophilic wire failed, we injected intravascular contrast media though the catheter, and if the contrast media could not reach the left atrium, PFO could not be diagnosed. If PFO could not be diagnosed, then pulmonary arteriovenous fistula (PAVF) was excluded by pulmonary angiography (Figure 4).

\section{Statistical Analysis}

Qualitative variables are presented as percentages and continuous data and expressed as the mean +- standard deviation. The chi-square test was used to compare the sensitivity and specificity of the three methods. AP value of $<0.05$ indicated statistical significance. All data were analyzed using SPSS software (version 18.0.1, SPSS Inc.).

\section{Results}

\section{Diagnosis of PFO}

Using the results of the right heart catheterization (RHC) as the gold standard for PFO diagnosis, 141 of the 161 patients studied were diagnosed with PFO, 18 patients could not be diagnosed with PFO, 2 patients were diagnosed with pulmonary arteriovenous malformation, and the morbidity of PFO was $88.17 \%$ (Table $1)$.

Diagnostic value of $c-T C D$

Two patients did not accept the c-TCD examination, right-to-left shunt was visualized at baseline by c-TCD in 97 patients $(70.28 \%, 97 / 138)$. Forty-four patients had mild RLS $(31.88 \%, 44 / 138), 22$ had moderate RLS $(15.94 \%, 22 / 138), 31$ had extensive RLS $(22.46 \%, 31 / 138)$. The Valsalva maneuver was effective in all c-TCD studies, and RLS was identified in 128 patients $(92.75 \%, 128 / 138)$. Thirty-six patients had mild RLS (26.09\%, 36/138), 28 had moderate RLS (20.29\%, 28/138), 64 had extensive RLS (46.38\%, 64/138). The difference between the baseline measurement and measurement during the performance of the Valsalva maneuver was statistically significant (Table 2).

Diagnostic value of c-TTE and c-TEE 
Loss of echo was identified in 12 patients $(8.51 \%, 12 / 141)$ by TTE. There were 3 patients with atrium septal aneurysms and 17 with color shunt $(12.06 \%, 17 / 141)$. Six patients did not agree to undergo the c-TTE examination, TEE bubbles were identified in 81 patients $(60.00 \%, 81 / 135)$ at baseline, including 41 with mild involvement $(30.37 \%, 41 / 135), 15$ with moderate involvemen $(11.11 \%, 15 / 135), 25$ with extensive involvemen $(18.52 \%, 25 / 135)$. The Valsalva maneuver was effective in all c-TTE studies, and RLS was identified 126 patients $(93.33 \%, 126 / 135), 24$ patients had mild RLS $(17.78 \%, 24 / 135)$, 35 patients had moderate RLS $(25.93 \%, 37 / 135)$, and 67 patients had extensive RLS $(49.63 \%, 67 / 135)$. Eight patients did not tolerate TEE examination, including seven with PFO and one without PFO. Two-dimensional TEE displayed a slit-like channel [?]2mm between the septum primum and the septum secundum in 119 patients (90.15\%, 119/132). Color shunt was identified in 40 patients $(30.30 \%, 40 / 132)$. Two patients did not undergo c-TEE examination, and TEE bubble was identified in 69 patients $(53.08 \%, 69 / 130)$ at baseline, 37 patients had mild results $(28.46 \%, 37 / 130), 12$ had moderate results $(9.23 \%, 12 / 130), 20$ had extensive results $(15.38 \%$, 20/130). The Valsalva maneuver was effective in all c-TEE studies, and RLS was identified 113 patients (86.92\%, 113/130), including 32 with mild RLS (24.62\%, 32/130), 15 with moderate RLS $(11.54 \%, 15 / 130)$, and 66 with extensive RLS (50.77\%, 66/130) (Table 2).

\section{Diagnosis of patients without PFO}

Of the total group of 161 patients, 18 patients could not be diagnosed with PFO. Among them, 4 (22.22\%, 4/18) patients had positive c-TCD both at baseline and with the Valsalva maneuver, and $7(38.89 \%, 7 / 18)$ patients had positive results only after the Valsalva maneuver was performed. Additionally, the c-TTE results for $6(33.33 \%, 6 / 18)$ patients were positive both at baseline and with the Valsalva maneuver, and 7 $(38.89 \%, 7 / 18)$ patients were positive only after the Valsalva maneuver was performed. The slit-like channel between the septum primum and the septum secundum on TEE was identified in $5(29.41 \%, 5 / 17)$ patients, and TEE bubble was identified in $4(23.53 \%, 4 / 17)$ patients both at baseline and with the Valsalva maneuver and in $6(35.29 \%, 6 / 17)$ patients only when the Valsalva maneuver was performed (Table 3$)$.

\section{Diagnostic value of the different methods}

In total, the sensitivity of c-TCD at baseline was $70.28 \%$ (97/138): 22.46\% (31/138) for extensive cases and $38.40 \%$ (53/138) for moderate and extensive cases. The sensitivity of c-TCD with the Valsalva maneuver was $92.75 \%$ (128/138): $46.38 \%(64 / 138)$ for extensive cases and $66.67 \%(92 / 138)$ for moderate and extensive cases. The sensitivity of TTE to loss of echo was $8.51 \%(12 / 141)$, and the sensitivity to color shunt was $12.06 \%(17 / 141)$. The sensitivity of the total c-TTE at baseline was $60.00 \%(81 / 135): 18.52 \%(25 / 135)$ for extensive cases and $29.63 \%$ (40/135) for moderate and extensive cases. The sensitivity of the total cTTE after the Valsalva maneuver was $93.33 \%(126 / 135)$ : $49.63 \%(67 / 135)$ for extensive cases and $75.56 \%$ $(102 / 135)$ for moderate and extensive cases. The sensitivity of TEE to the slit-like channel between the septum primum and the septum secundum was $90.15 \%$ (119/132), the sensitivity to color shunt was $30.30 \%$ (40/132); the sensitivity of the total c-TEE at baseline was 53.08\% (69/130): 15.38\% (20/130) for extensive cases and $24.62 \%(32 / 130)$ for moderate and extensive cases. The sensitivity of the c-TEE after the Valsalva maneuver was $86.92 \%$ (113/130): $50.77 \%$ (66/130) for extensive cases and $62.31 \%$ (81/130)for moderate and extensive cases.

In the comparison among groups, there were no significant difference between the sensitivity of total c-TCD, c-TTE and c-TEE at baseline. When pairs of methods were compared, c-TCD was significantly more sensitive than c-TEE $(P=0.004)$. With the Valsalva maneuver, the sensitivity of the total c-TTE was significantly higher than that of c-TEE $(P=0.041)$. For the within-group comparisons, when bubbles were extensive in c-TTE examination at baseline, c-TTE had significantly higher sensitivity than c-TCD $(P=0.011)$ and c-TEE $(P=0.009)$.

The positive predictive value of total c-TCD at baseline was 96.04\% (97/101): 96.88\% (31/32) for extensive cases and $98.15 \%(53 / 54)$ for moderate and extensive cases. With the Valsalva maneuver, the positive predictive value of total c-TCD was $92.09 \%$ (128/139): $96.97 \%$ (64/66) for extensive cases and $96.84 \%$ (92/95) for moderate and extensive cases. The false negative rate of total c-TCD was $29.71 \%(41 / 138)$ at baseline and 
$7.25 \%(10 / 138)$ after the Valsalva maneuver. The positive predictive value of c-TTE at baseline was $93.10 \%$ (81/87): $100.00 \%$ (25/25) for extensive cases and 97.56\% (40/41) for moderate and extensive cases. After the Valsalva maneuver, the positive predictive value was $90.65 \%$ (126/139): $97.10 \%(67 / 69)$ for extensive cases and $93.58 \%$ (102/109) for moderate and extensive cases. The false negative rate of total c-TTE was $40.00 \%$ $(54 / 135)$ at baseline and $6.67 \%(9 / 135)$ after the Valsalva maneuver. The positive predictive value of c-TEE at baseline was $94.52 \%$ (69/73): $95.24 \%$ (20/21) for extensive cases and 96.97\% (32/33) for moderate and extensive cases. After the Valsalva maneuver, the positive predictive value was $91.87 \%$ (113/123): $95.65 \%$ $(66 / 69)$ for extensive cases and $93.10 \%(81 / 87)$ for moderate and extensive cases. The false negative rate of total c-TEE was $46.92 \%(61 / 130)$ at baseline and $13.08 \%$ (17/130) after the Valsalva maneuver (Table 4).

\section{Discussion}

Foramen ovale is a persistent fetal communication between the right and left atrium caused by the incomplete closure of the atrial septum. After birth, the foramen ovale normally closes, but the morbidity of PFO was about $30 \%{ }^{[8]}$. PFO normally has no obvious symptoms, it is difficult to hear an associated heart murmur, and there are no abnormalities on electrocardiogram or chest X-ray, therefore, PFO is easy to overlook. It was recently proposed that for patients who suffer from migraine headaches, cryptogenic stroke, TIA, and cerebral infarction that do have acute cerebral infarction or anterior circulation transient ischemic attack on admission, the possibility of right-to-left shunt as a cause should be considered.

Our study assessed a large series of patients to identify the presence of right-to-left shunt. The results demonstrated that c-TTE has better sensitivity than c-TEE in the diagnosis of PFO both at baseline and with the Valsalva maneuver; c-TCD performed similarly to c-TEE but maybe produce more false positives. TEE with contrast showed similar sensitivity to c-TCD, but had a higher probability of false-negatives, therefor, c-TCD can be used as an effective supplement for diagnosing PFO. TEE had the highest sensitivity of the three methods and can be considered the gold standard. However, the results of c-TCD can only point out the presence of right-to-left shunt and cannot exclude extracardiac shunt, so additional measures should be used.

PFO is a three-dimensional structure that changes shape with the cardiac cycle. Therefore, it is difficult to fully display a PFO using TEE's two-dimensional technology, and in our results, there were some false positive cases when we used 2D TEE images ( 5 cases). The use of three-dimensional (3D) technology can solve the one-sidedness problem, but the quality of 3D images can be poor compared to that of 2D images; sometimes, 3D images were only used for reference ${ }^{[9]}$. In terms of sensitivity, total c-TTE after the Valsalva maneuver had the highest sensitivity, especially for extensive cases, we consider that sensitivity increased with increasing quantities of bubbles in the left heart. Moreover, when we observed color shunt from the slit-like channel between the septum primum and the septum secundum on TEE, the positive predictive value was $100.00 \%$; therefor, in our daily clinical work, the observation color shunt on TEE is very important. However, the sensitivity of c-TEE is lower than that of c-TTE after the Valsalva maneuver, based on our observations, patients sometimes cannot perform the Valsalva maneuver perfectly during TEE examination, which is the most likely reason for the reduced sensitivity during the Valsalva maneuver. c-TCD has the greatest value in diagnosing PFO; almost all the PFO patients had positive results on the c-TCD examination, but cTCD can only identify right-to-left shunt and cannot exclude extracardiac shunt ${ }^{[10]}$; c-TTE and c-TEE can observe the structure of the foramen ovale and are more convincing. The positive predictive value can reflect the possible prevalence rate of patients with positive results. For all the three methods (c-TCD, c-TTE, c-TEE), when there were a large number of bubbles in the left heart, especially in the presence of color shunt from the slit-like channel, the positive predictive value was the highest. Although c-TCD had the highest positive predictive value, some unsolved problems remained; for example, some bubbles can get though the pulmonary circulation and be detected in the cerebral arteries, leading to a false positive result. On the other hand, c-TTE and c-TEE had more false negatives, and the rate of misdetection might be higher than that for c-TCD. Thus, for suspected patients, c-TCD should be performed first; if positive results are obtained, then c-TTE and c-TEE should be used for further confirmation.

The results sometimes were different between the resting state and the Valsalva maneuver, and the positive 
rate was higher with the Valsalva maneuver. In the resting state, RLS could not be observed in all the patients. In some PFO patients, the foramen ovale is closed in resting state, only under some particular situation, for example, cough, cry, constipation, Valsalva maneuver and so on, that's also the causes of nervous system symptoms on PFO patients ${ }^{[11]}$. Only under those situations, the right atrial pressure can higher than the left atrial transiently, the foramen ovale then opened, so we could observe RLS. Therefore, when no bubbles were present in the left heart, the Valsalva maneuver was necessary, but the results were positive with or without the Valsalva maneuver when bubbles were present in left heart.

Pulmonary arteriovenous fistula (PAVF) is an abnormal pulmonary vascular structure that connects a pulmonary artery to a pulmonary vein, bypassing the normal capillary bed and resulting in an intrapulmonary right-to-left shunt ${ }^{[12]}$. In our study, 2 patients were diagnosed with PVAF. At first, not much attention was paid to this disease; however, because the results of c-TCD, c-TTE and c-TEE were strongly positive, we performed the transcatheter examination, and unexpectedly, PFO could not be diagnosed. Pulmonary angiography was then performed and the results showed that the contrast medium could get to the pulmonary vein from the pulmonary artery, indicating that we had previously made a diagnostic mistake. When we reviewed the examinations, we found that the slit-like channel on TEE was not very clear, and the bubbles seemed to come from the roof or the entrance of the pulmonary vein; these findings could help to diagnose PVAF. Therefore, in conclusion, we must consider the following points when diagnosing PVAF: first, the results of c-TCD, c-TTE and c-TEE show a positive and sometimes strong reaction; second, TEE images cannot account for the existence of the slit-like channel, or the width of foramen ovale does not match the quantity of bubbles; third, the bubbles seemed to come from the roof, the entrance of pulmonary vein, and not from the foramen ovale.

In conclusion, the present study reveals that c-TTE with the Valsalva maneuver yields a higher sensitivity in detecting PFO-RLS, but the rate of misdetection might be higher than that of c-TCD. Therefore, patients with suspected PFO, can be examined with c-TCD first, and if positive results are obtained, then c-TTE and c-TEE should performed for further confirmation. This procedure may be helpful for the diagnosis of patent foramen ovale in practice.

\section{Funding}

The research reported in this article was supported by the technology capture project of Shandong Province under award number 2017GGX90104.

\section{References}

[1]Robert G Hart, Luciana Catanese, Kanjana S Perera, George Ntaios, Stuart J Connolly . Embolic Stroke of Undetermined Source: A Systematic Review and Clinical Update. Stroke. 2017 Apr;48(4):867-872.

[2]Mohammad K Mojadidi, Muhammad O Zaman, Islam Y Elgendy, Ahmed N Mahmoud, Nimesh K Patel, Nayan Agarwal, Jonathan M Tobis, Bernhard Meier. Cryptogenic Stroke and Patent Foramen Ovale. J Am Coll Cardiol. 2018 Mar 6;71(9):1035-1043.

[3]Rubeena Khan, Anthony K Chan, Tapas K Mondal, Bosco A Paes, Thrombosis and Hemostasis in Newborns (THIN) Group. Patent Foramen Ovale and Stroke in Childhood: A Systematic Review of the Literature. Eur J Paediatr Neurol 2016 Jul;20(4):500-11.

[4]David Hildick-Smith, Timothy M Williams . Patent Foramen Ovale and Migraine Headache. Interv Cardiol Clin 2017 Oct;6(4):539-545.

[5]Yusuf Ali Kausar Rushdi, Hina Hina, Bijendra Patel, Frank W Cross. The Incidence of Peripheral Arterial Embolism in Association With a Patent Foramen Ovale (Right-To-Left Shunt). JRSM Short Rep 2011 May;2(5):35

[6]Gramiak R, Shah PM, Kramer DH. Ultrasound cardiography: contrast studies in anatomy and function. Radiology. 1969 Apr;92(5):939-48. 
[7]E K Kerut, W T Norfleet, G D Plotnick, T D Giles. Patent Foramen Ovale: A Review of Associated Conditions and the Impact of Physiological Size. J Am Coll Cardiol. 2001 Sep;38(3):613-23.

[8]Shunichi Homma, Steven R Messe, Tatjana Rundek, Yee-Ping Sun, Jennifer Franke, Karina Davidson, Horst Sievert, Ralph L Sacco, Marco R Di Tullio. Patent Foramen Ovale. Nat Rev Dis Primers. 2016 Jan 21; 2:15086.

[9]Miriam Shanks, Dulka Manawadu, Isabelle Vonder Muhll, Khurshid Khan, Harald Becher, Jonathan Choy. Detection of Patent Foramen Ovale by 3D Echocardiography. JACC Cardiovasc Imaging. 2012 Mar;5(3):32931.

[10]Benjamin S Wessler, David M Kent, David E Thaler, Robin Ruthazer, Jennifer S Lutz, Joaquin Serena. The RoPE Score and Right-to-Left Shunt Severity by Transcranial Doppler in the CODICIA Study. Cerebrovasc Dis. 2015;40(1-2):52-8.

[11]Enfa Zhao, Yajuan Wei, Yafei Zhang, Nina Zhai, Ping Zhao, Baomin Liu. A Comparison of Transthroracic Echocardiograpy and Transcranial Doppler With Contrast Agent for Detection of Patent Foramen Ovale With or Without the Valsalva Maneuver Medicine (Baltimore).2015 Oct;94(43):e1937.

[12]Ahmed Kamel Abdel Aal, Joseph Eason, Sherif Moawad, Khalid Mahmoud, Basant Farouk Hamed, Moustafa Omar Massoud, Nathan Ertel, Andrew Gunn, Rachel Oser, Souheil Saddekni. Persistent Pulmonary Arteriovenous Malformations: Percutaneous Embolotherapy. Curr Probl Diagn Radiol. 2018 Nov;47(6):428-436.

Table 1:Baseline characterization of patients

\begin{tabular}{ll}
\hline Items & Cases \\
\hline Total cases & 161 \\
Male & 86 \\
Famale & 75 \\
Age(years) & $42.0 \pm 15.6$ \\
migraine & 55 \\
cryptogenic stroke (CS) & 56 \\
migraine +CS & 20 \\
TIA & 9 \\
dizzy or syncope & 19 \\
epilepsy & 2 \\
Diagnosed of PFO & 141 \\
\hline
\end{tabular}

Table 2, Results of c-TCD, c-TTE and c-TEE in 141 cases

\begin{tabular}{|c|c|c|c|c|}
\hline & & & Baseline & $\begin{array}{l}\text { Valsalva } \\
\text { maneuver }\end{array}$ \\
\hline \multirow[t]{4}{*}{ c-TCD* n $=138$} & $\mathrm{c}-\mathrm{TCD}^{*} \mathrm{n}=138$ & negative & 41 & 10 \\
\hline & & mild & 44 & 36 \\
\hline & & moderate & 22 & 28 \\
\hline & & extensive & 31 & 64 \\
\hline \multirow[t]{4}{*}{ c-TTE\# $\mathrm{n}=135$} & negative & negative & 54 & 9 \\
\hline & mild & mild & 41 & 24 \\
\hline & moderate & moderate & 15 & 35 \\
\hline & extensive & extensive & 25 & 67 \\
\hline $\begin{array}{l}\text { TTE 2D }(+), \\
\mathrm{n}=141\end{array}$ & $\begin{array}{l}\text { TTE 2D }(+), \\
\mathrm{n}=141\end{array}$ & $\begin{array}{l}\text { TTE 2D }(+), \\
\mathrm{n}=141\end{array}$ & 12 & \\
\hline
\end{tabular}




\begin{tabular}{|c|c|c|c|c|}
\hline & & & Baseline & $\begin{array}{l}\text { Valsalva } \\
\text { maneuver }\end{array}$ \\
\hline $\begin{array}{l}\text { TTE color }(+), \\
\mathrm{n}=141\end{array}$ & $\begin{array}{l}\text { TTE color }(+), \\
\mathrm{n}=141\end{array}$ & $\begin{array}{l}\text { TTE color }(+), \\
\mathrm{n}=141\end{array}$ & 17 & \\
\hline \multirow[t]{4}{*}{$c-T E E \& n=130$} & $\mathrm{c}-$ TEE\& $\mathrm{n}=130$ & negative & 61 & 17 \\
\hline & & mild & 37 & 32 \\
\hline & & moderate & 12 & 15 \\
\hline & & extensive & 20 & 66 \\
\hline $\begin{array}{l}\text { TEE 2D }(+), \\
\mathrm{n}=132\end{array}$ & $\begin{array}{l}\text { TEE 2D }(+), \\
n=132\end{array}$ & $\begin{array}{l}\text { TEE 2D }(+), \\
\mathrm{n}=132\end{array}$ & 119 & \\
\hline $\begin{array}{l}\text { TEE color }(+), \\
n=132\end{array}$ & $\begin{array}{l}\text { TEE color }(+), \\
\mathrm{n}=132\end{array}$ & $\begin{array}{l}\text { TEE color }(+), \\
\mathrm{n}=132\end{array}$ & 40 & \\
\hline
\end{tabular}

*: Two patients did not accept the c-TCD examination; \#: Six patients did not accept the c-TTE examination; \&:Seven patients did not accept the TEE examination.

Table 3 Diagnosis of patients without PFO (18 cases)

\begin{tabular}{llll}
\hline & & Baseline & Valsalva maneuver \\
\hline c-TCD & negative & 14 & 7 \\
& mild & 3 & 8 \\
& moderate & 0 & 1 \\
c-TTE & extensive & 1 & 2 \\
& negative & 12 & 5 \\
& mild & 5 & 6 \\
TTE 2D $(+)$ & moderate & 1 & 5 \\
TTE color $(+)$ & extensive & 0 & 2 \\
c-TEE* & TTE 2D $(+)$ & 0 & 0 \\
& negative & 13 & 0 \\
& mild & 3 & 4 \\
TEE 2D $(+)$ & moderate & 0 & 3 \\
TEE color $(+)$ & TEtensive & 1 & 3 \\
& TEE color $(+)$ & 5 & 5 \\
\end{tabular}

*: One of the 18 patients did not tolerate TEE.

Table 4, Diagnostic value of c-TCD, c-TTE and c-TEE

\begin{tabular}{|c|c|c|c|c|c|c|c|c|}
\hline & & & Baseline & Baseline & & $\begin{array}{l}\text { Valsalva } \\
\text { maneuver }\end{array}$ & $\begin{array}{l}\text { Valsalva } \\
\text { maneuver }\end{array}$ & \\
\hline \multirow{4}{*}{ c-TCD } & & & Sensi $(\%)$ & $\operatorname{PPV}(\%)$ & $\operatorname{FNR}(\%)$ & Sensi $(\%)$ & $\operatorname{PPV}(\%)$ & $\overline{\text { FNR }(9}$ \\
\hline & c-TCD & Total & $70.29^{* *}$ & 96.04 & 29.71 & 92.75 & 92.09 & 7.25 \\
\hline & & extensive & 22.46 & 96.88 & 56.94 & 46.38 & 96.97 & 13.51 \\
\hline & & $\begin{array}{l}\text { moderate } \\
+ \\
\text { extensive }\end{array}$ & 38.40 & 98.15 & 43.62 & 66.67 & 96.84 & 9.80 \\
\hline c-TTE & Total & Total & 60.00 & 93.10 & 40.00 & $93.33^{*}$ & 90.65 & 6.67 \\
\hline
\end{tabular}




\begin{tabular}{|c|c|c|c|c|c|c|c|c|}
\hline & & & Baseline & Baseline & & $\begin{array}{l}\text { Valsalva } \\
\text { maneuver }\end{array}$ & $\begin{array}{l}\text { Valsalva } \\
\text { maneuver }\end{array}$ & \\
\hline & extensive & extensive & 18.52 & $100 \#$ & 68.35 & 49.63 & 97.10 & 11.84 \\
\hline & moderate & moderate & 29.63 & 97.56 & 57.44 & 75.56 & 93.58 & 8.10 \\
\hline & & & & & & & & \\
\hline & extensive & extensive & & & & & & \\
\hline TTE 2D & TTE 2D & TTE 2D & 8.51 & 100 & & & & \\
\hline TTE & TTE & TTE & 12.06 & 100 & & & & \\
\hline color & color & color & & & & & & \\
\hline \multirow[t]{4}{*}{ c-TEE } & c-TEE & Total & 53.08 & 94.52 & 46.92 & 86.92 & 91.87 & 13.08 \\
\hline & & extensive & 15.38 & 95.24 & 75.30 & 50.77 & 95.65 & 20.48 \\
\hline & & $\begin{array}{l}\text { moderate } \\
+\end{array}$ & 24.62 & 96.97 & 65.59 & 62.31 & 93.10 & 17.35 \\
\hline & & extensive & & & & & & \\
\hline TEE 2D & TEE 2D & TEE 2D & 90.15 & 94.17 & 9.85 & & & \\
\hline TEE & TEE & TEE & 30.30 & 100 & & & & \\
\hline color & color & color & & & & & & \\
\hline
\end{tabular}

PPV: positive predictive value; Sensi: Sensitive; FNR: false negative rate. ${ }^{* *}: P$ ¡0.005(0.004) vs c-TEE; *: $P \nmid 0.05(0.041)$ vs c-TEE; \#: $P$ ¡0.05(0.011) vs extensive of c-TCD, (0.009) vs extensive of c-TEE.

FIGURE 1. Quantification of the shunt by c-TCD. (A) Grade I, mild signals. (B) Grade II, moderate signals. (C) Grade III, extensive signals.

FIGURE 2. Quantification of the shunt by c-TTE. (A) Grade I, mild signals. (B) Grade II, moderate signals. (C) Grade III, extensive signals. LA: left atrium; LV: left ventricle; RA: right atrium; RV: right ventricle.

FIGURE 3. Two-dimensional and color Doppler TEE; quantification of the shunt by c-TTE. (A) Twodimensional TEE displayed the slit-like channel between the septum primum and the septum secundum. (B) Color Doppler displayed right-to-left color shunting. (C) Grade I, mild signals. (D) Grade II, moderate signals. (E) Grade III, extensive signals. LA: left atrium; LV: left ventricle; RA: right atrium; RV: right ventricle.

FIGURE 4. Right heart catheterization with angiography. (A) After intravascular contrast media was injected though the catheter, if the contrast media cannot reach the left atrium, PFO cannot be diagnosed. (B \& C) If PFO cannot be diagnosed, then pulmonary arteriovenous fistula (PAVF) is excluded by pulmonary angiography. (D) Pulmonary arteriography showed PAVF.

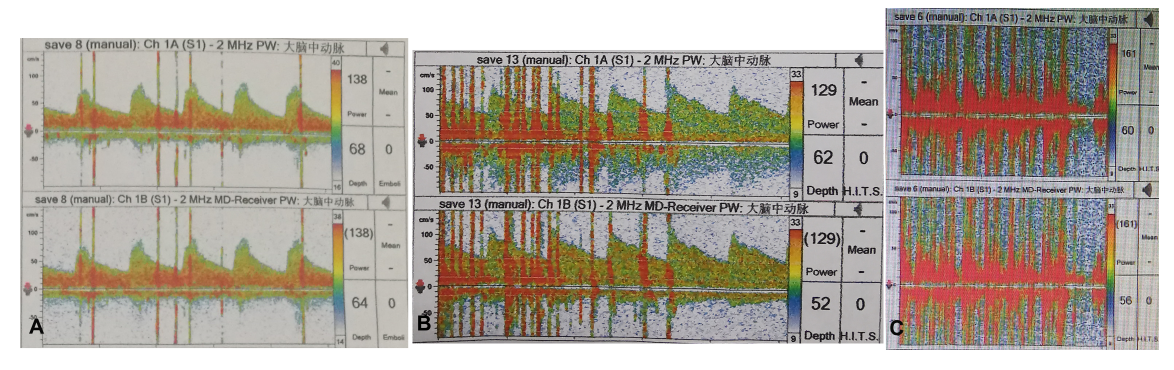



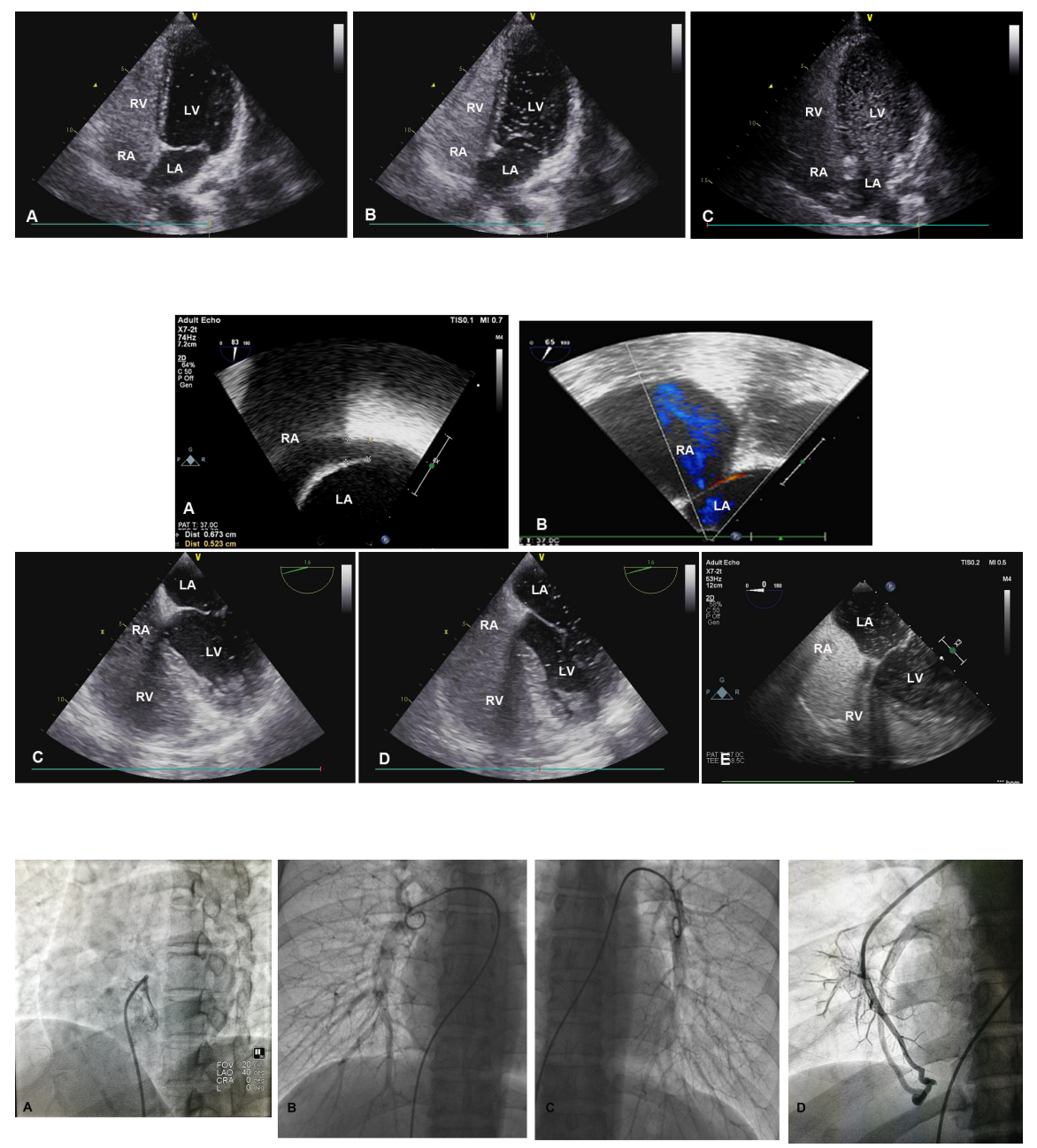